\title{
NEUTRON REFLECTOMETRY STUDIES OF THIN FILMS AND MULTILAYERED MATERIALS
}

\author{
C.F. Majkrzak \\ Center for Neutron Research, National Institute of Standards \& Technology \\ Gaithersburg, Maryland, 20899, USA
}

\begin{abstract}
Neutron reflectometry is an important technique for studying the composition and structure of thin films and layered media on a nanometer scale. Neutrons are particularly useful as probes of organic and magnetic materials since the information that can be obtained is often unique. Furthermore, the fact that neutrons traverse relatively large distances in single crystalline materials, such as silicon, permit investigations in diverse sample environments. The fundamental theoretical principles and experimental methodology of neutron reflectometry are presented, in addition to several illustrative examples of measurements relevant to polymer science, biology, electrochemistry, and magnetism.
\end{abstract}

PACS numbers: $61.12 .-\mathrm{q}$

\section{Introduction}

The intensity of a neutron beam reflected at a glancing angle from a flat material surface depends upon the composition and structure of the underlying matter. More specifically, if the reflected intensity is measured at an angle of reflection equal to that of incidence, known as the specular condition, then the reflectivity, or ratio of reflected to incident intensity, as a function of glancing angle, can be analyzed to reconstruct an averaged compositional depth profile along the normal to the surface. This is illustrated schematically in Fig. 1. At sufficiently low angles, a given slab of thickness $\mathrm{d} z$ can be represented by a single uniform scattering potential or density that is proportional to the sum of the products of each constituent atom's scattering power (characterized by a scalar scattering length) and its number per unit volume. The depth profile depicted in Fig. 1 represents the average values of the scattering density for the slabs along the direction of the surface normal, as can be obtained from the measured reflectivity curve. The spatial resolution, or minimum slab thickness, is inversely proportional to the angular range over. which the reflectivity is measured and is of the order of nanometers in typical experiments. Knowing the scattering power associated with a particular atom or isotope, the chemical compositional profile can be deduced. This picture 


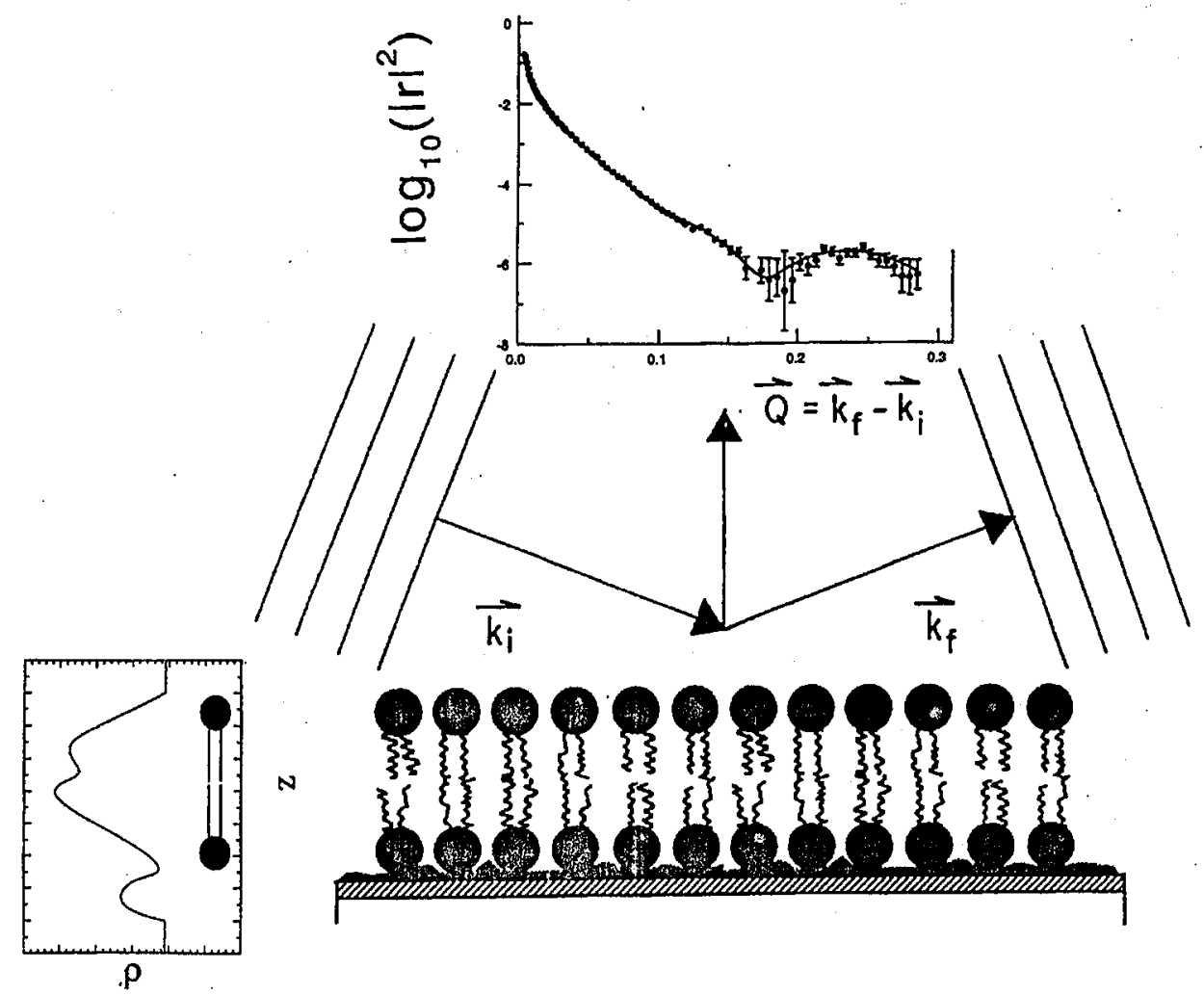

Fig. 1. Schematic representation of specular reflection at glancing angles of incidence as described in the text. The SLD profile along the surface normal is deduced from the reflected intensities measured as a function of wave vector transfer.

of the distribution of matter normal to the surface is nonlocal in the sense that the dimensions of the in-plane or latteral area over which the neutron wave effectively averages is relatively large compared to the thickness of the slabs. This is characteristic of diffraction measurements in general and distinguishes reflectivity from more spatially local probes such as atomic force and tunnelling electron microscopies. It should be mentioned, nonetheless, that in-plane variations of the scattering density, including possible correlations, are manifested directly in nonspecular scattering where the angles of incidence and reflection are chosen not to be equal. Although nonspecular scattering is of significant importance and interest in the study of thin films, it is largely beyond the scope of this paper which is concerned primarily with specular measurements. The interested reader should refer to the literature on nonspecular scattering [1].

For neutrons, the sensitivity for distinguishing different elements from one another depends on the contrast in scattering potentials which are predominantly nuclear in origin and, consequently, isotope specific. For hydrogen and deuterium, that contrast is relatively large: therefore, neutron reflectivity is particularly use- 
ful for studying soft condensed matter systems, including polymer and LangmuirBlodgett films and also biological membranes. This isotopic specificity complements the $Z$-dependent interaction of X-rays with atomic electrons which determines the sensitivity for X-ray reflectometry - often both radiations are valuable in the study of certain systems. In addition, the interaction between the magnetic moment of the neutron and that of an atom depends i:pon the relative orientation of the moments, and can have a strength comparable to that of the nuclear interaction. Using polarized neutron beams, it is in fact possible to obtain the vectorial magnetization depth profile in films and layered structures.

Another advantageous property is the neutron's high transmission through single-crystalline materials, such as silicon, which allows the substrate supporting the film of interest to also serve as incident medium: hence, for example, films in contact with nontransparent liquid reservoirs can be studied. Furthermore, the flat and smooth surface of a substrate can be employed, in some cases, as a coherent support structure on which molecules can be made to self-assemble with a common axis of orientation. Specular reflection measurements then effectively yield the chemical profile of the unit molecule along the surface normal direction.

Sections 2 and 3 discuss, in more detail, elements of the theory and practice of neutron reflectometry, respectively. Section 4 presents a number of illustrative examples of the use of neutron reflectivity measurements in materials science. Section 5 considers phase determination and inversion of reflectivity data to obtain the scattering density profile directly.

\section{Fundamental theory}

\subsection{Chemical structures}

An important distinction between neutron or X-ray scattering and electron and atomic or molecular beam diffraction is the simplicity with which the former fundamental interactions can be described. The neutron reflection amplitude, $r$, from a nonmagnetic layered structure with a surface of infinite lateral extent, and for the specular condition depicted in Fig. 1, is given by

$$
\left.r=\frac{4 \pi}{\mathrm{i} Q} \int_{-\infty}^{+\infty} \Psi \rho(z)\right) \mathrm{e}^{\mathrm{i} Q z / 2} \mathrm{~d} z .
$$

In Eq. (1) $\Psi$ represents the wave function within the scattering medium at a depth $z$ below the bounding surface, $Q$ is the magnitude of the wave vector transfer $k_{\mathrm{f}}-k_{\mathrm{i}}$, and $\rho$ is the scattering length density (SLD) which is the sum of the products of a scalar coherent scattering length and number of scattering centers per unit volume for each isotope present. The validity of this expression depends upon the assumptions that the incident neutron can be represented by a plane wave, the scattering is both specular and elastic, and the nuclear spins are randomly oriented in space - requirements which are satisfied in most cases of interest. In addition, it is implicitly assumed that the reflection occurs at sufficiently low glancing angles that the scattering medium can be treated as a continuum without any effects of crystalline order being manifested (at higher angles, the integral over the continuum must be replaced by a summation on discrete atomic planes: see Ref. [2] for a more complete discussion). 
At sufficiently high glancing angles, but still within the continuum limit, where the scattering is weak enough, Eq. (1) can be simplified even further by imposing the well-known Born approximation that the wave function $\Psi$ within the medium can be replaced by a form equivalent to an undistorted plane wave in vacuum. This approximation is almost always used in neutron and X-ray scattering from polycrystalline or powder samples. Then, the reflection amplitude is directly related to the SLD by a Fourier transform. Unfortunately, the approximation breaks down at lower reflection angles for most film systems of interest, either because of the strength of the scattering from the film itself or from the substrate on which the film has been deposited. This is illustrated in Fig. 2 which compares the prediction of Eq. (1) in the Born approximation with that obtained according to a more accurate solution to be discussed below. Although the apparently significant discrepancies in the two curves occur over a limited range at the very lowest angles, where the reflectivity, $|r|^{2}$, approaches unity, this is precisely where some of the most important information regarding the SLD profile is found.

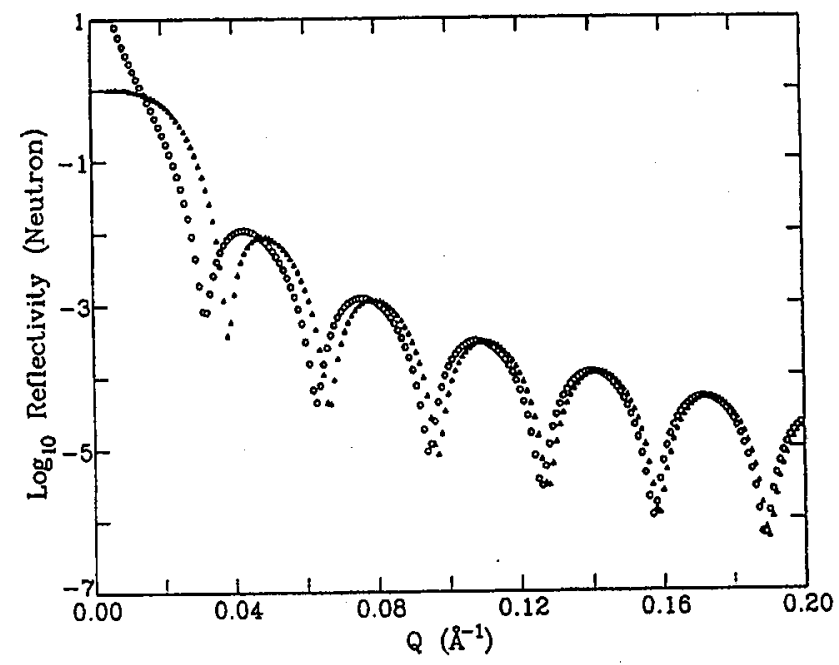

Fig. 2. Reflectivity curves calculated according to the Born approximation (circles) and the more accurate theory (triangles) discussed in the text. $200 \AA \mathrm{Ni}$, n Si substrate.

It is of no small consequence, then, that the "exact" expression for the reflection amplitude given by Eq. (1) can, in fact, be solved straightforwardly, without any need to make the Born approximation, via a simple differential wave equation. One of the most basic problems in quantum mechanics is the description of the reflection and transmission of a plane wave by a rectangular potential barrier in one dimension. It turns out that this is equivalent to the problem at hand for analyzing neutron specular reflection. By imposing the boundary conditions that the wave function and its first derivative be continuous at each boundary, the conditions which ensure that particle number and momentum are conserved, the solution of the wave equation (see, for example, Ref. [3]) 


$$
\frac{\mathrm{d}^{2} \Psi}{\mathrm{d} z^{2}}+\left(\frac{Q}{2}\right)^{2} \Psi=0
$$

leads to a set of simultaneous algebraic equations relating the reflected and transmitted amplitudes, $r$ and $t$, respectively, to that of the incident wave of unit value. These equations are compactly represented in matrix form as

$$
\left(\begin{array}{c}
t \\
\mathrm{i} n_{\mathrm{b}} t
\end{array}\right)=\left(\begin{array}{cc}
A & B \\
C & D
\end{array}\right)\left(\begin{array}{c}
1+r \\
\mathrm{i} n_{\mathrm{f}}(1-r)
\end{array}\right)
$$

where

$$
n_{\mathrm{b}(\mathfrak{f})}^{2}=1-\frac{16 \pi \rho_{\mathrm{b}(\mathfrak{f})}}{Q^{2}}
$$

and the subscripts on $n$ refer to the potential in front (f) or in back (b) of the rectangular potential barrier of height $\rho$ and thickness $\Delta z$. The elements $A, B, C$, and $D$ of the transfer matrix contain all of the information about the height and width of the barrier. Furthermore, any arbitrary SLD profile can be represented to any degree of accuracy by division into a sufficient number of adjacent rectangular slabs of constant SLD. The transfer matrix describing this more complicated composite structure can be written as the product of all the individual slab matrices. The details of this piecewise continuous treatment are discussed in Refs. [2] and [4].

The matrix formulation described above for relating the reflection amplitude to the SLD profile has proven to be remarkably accurate in the analysis of actual neutron reflectivity measurements for samples which have relatively smooth interfaces and do not give rise to appreciable nonspecular or diffuse scattering. At this point it should be emphasized that it is the square of the complex reflection amplitude $r$ that is measured in experiment; i.e., the ratio of the reflected to incident beam intensities. $|r|^{2}$ can be calculated from a model structure using Eq. (3) for either predicting what reflectivity should be expected in an experimental measurement, or for use in nonlinear least squares fitting routines [5] as well as model independent procedures [ 6$]$ for interpreting reflectivity data. However, obtaining accurate and unambiguous SLD profiles from measured reflectivity data is beset with a number of fundamental difficulties. First of all, measuring $|r|^{2}$ rather than the amplitude $r$ results in a complete loss of the phase information that is essential for establishing uniqueness; i.e., more than one potential can give rise to the same reflectivity curve. The phase problem is discussed in Sec. 5 below. Secondly, fitting reflectivity data to extract a SLD profile is a highly nonlinear process. To add to these complications is the reality that reflectivity data can be collected only over a finite range of scattering angle, thereby introducing, to some degree, truncation artifacts in the SLD profile. Fortunately, in many cases these potentially significant problems can be effectively minimized with proper experimental technique and data analysis.

\subsection{Magnetic materials and polarized neutrons}

As already mentioned in the Introduction, the interaction between the magnetic moments of a neutron and an atom depends upon their relative orientation and can be comparable in strength to that arising from the nuclear potential 


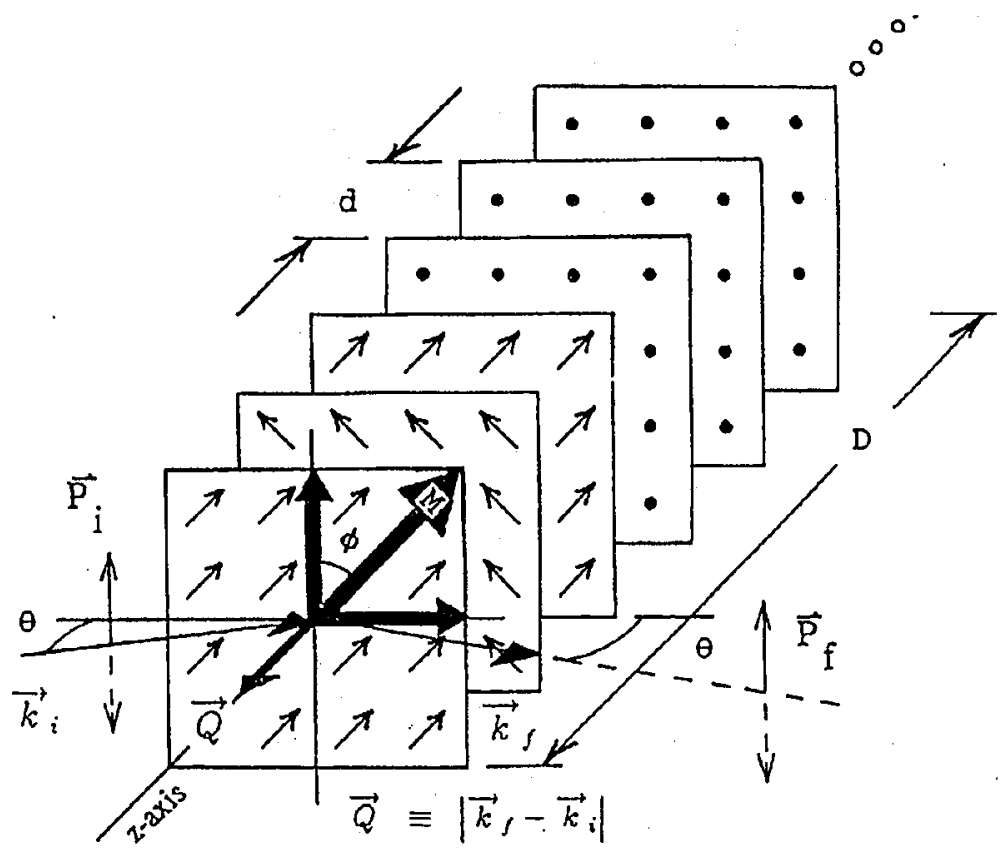

Fig. 3. Diagram representing successive layers with noncollinear magnetizations. Polarized neutron beams can be used to determine the in-plane vector magnetization depth profile along the surface normal.

(see [7] for a more detailed account). Because of the spin-dependent nature of the interaction; a vector magnetization depth profile along the surface normal can be obtained for a magnetic thin film structure using polarized neutron beams. Four independent reflectivities can be measured, two involving no reversal or "flip" of the neutron spin eigenstate and two resulting in a change of state from either " + " to "-" or vice versa. For the specific scattering geometry illustrated in Fig. 3, the component of the net atomic magnetization in a given slab which is parallel to the neutron polarization $P$ can contribute only to non-spin-flip (NSF) scattering while the perpendicular magnetization component gives rise solely to spin-flip (SF) processes. Further, the NSF reflectivities involve an interference between magnetic and nuclear potentials whereas the SF reflectivities are purely magnetic in origin. Because of the possibility of spin-flip, the polarized beam version of Eq. (3) for the reflection amplitude requires 4 -component vectors and a $4 \times 4$ transfer matrix. A more complete discussion is given, for example, in Ref. [2].

\section{Experimental methods}

Figure 4 is a schematic representation of one of the two reflectometers at NIST. The pairs of masks preceding and following the sample define a ribbon-like beam with relatively narrow angular divergence in the scattering plane defined by the incident and reflected wave vectors. The vertical divergence, on the other hand, is relatively large, of the order of 2.5 degrees, but does not significantly affect 
the instrumental resolution $\mathrm{d} Q / Q$. For specular reflectometry, reflected intensities are measured as a function of the glancing angle, defined by the incident beam direction and the surface of the sample. In this mode, the detector is positioned at twice the glancing angle. Because the incident beam is nearly monochromatic, each scattering angle corresponds to a single nominal value of the wave vector transfer $Q$. At pulsed neutron sources, polychromatic beams are used at fixed. scattering angles: a given neutron wavelength, identified by its time of flight, then corresponds to a particular value of $Q$. In the continuous source configuration, the footprint of the beam incident on the sample and the instrumental resolution can be kept approximately constant by increasing the slit apertures with angle (a typical value of $\mathrm{d} Q / Q$ is 0.025 ).

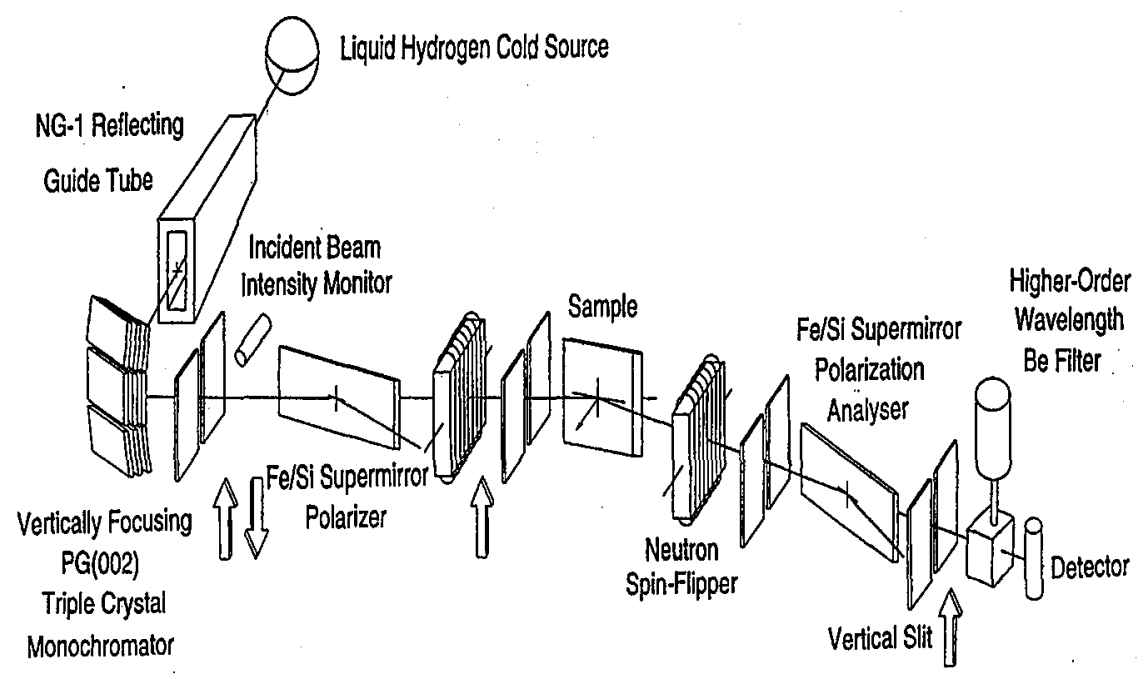

Fig. 4. Polarized beam neutron reflectometer schematic.

The transmission polarizers depicted in Fig. 4 are described in Ref. [8] and have polarizing efficiencies in excess of $95 \%$ and transmission losses for the desired spin state of only about $20 \%$. The polarizers can transmit beams up to approximately $0.6 \mathrm{~cm}$ wide and are of sufficient height to accept the full vertical dimension of the beam which is focussed down to about $3 \mathrm{~cm}$ at the sample position. The flat electromagnetic coil flippers allow highly efficient rotation of one neutron spin state to the other in both incident and reflected beams.

The instrumental $Q$-resolution in neutron reflectometry can be often tightened sufficiently to resolve the oscillation fringes observed in the reflectivity curve measured for a simple homogeneous thin film up to about $5000 \AA$ thick without prohibitive loss of intensity. (Fringes from a thimner, not quite uniform film are. evident in Fig. 5.) The overall thickness of a film with relatively abrupt interfaces can in many cases be determined with a fractional accuracy of the order of a few per cent or better because of the sensitivity to the positions of sharp minima in the reflectivity fringes. Clearly, if the substrate which supports a film of interest 


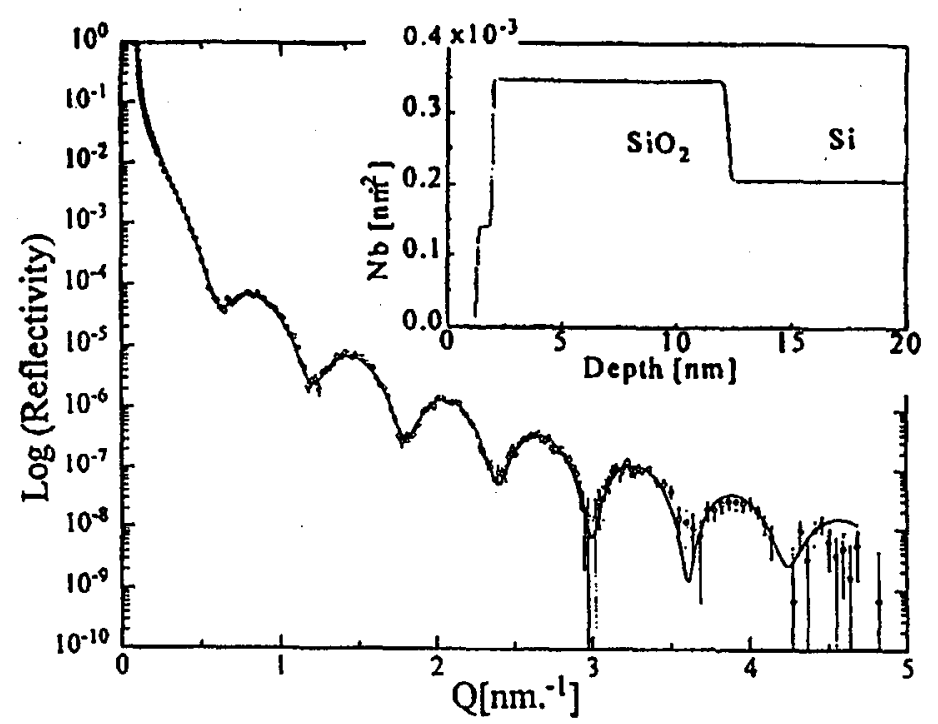

Fig. 5. Neutron reflectivity curve obtained for an oxide film on the surface of a single crystalline Si substrate. The SLD profile corresponding to the fit (solid line) to the data (symbols) is shown in the inset.

is not perfectly flat but curved, particularly if the radius of curvature lies in the scattering plane defined by the incident and reflected wave vectors, then the effective angular divergence of the incident beam will be increased, resulting in reduced $Q$-resolution.

In contrast, the spatial resolution associated with the more detailed features which may occur in a particular SLD profile is inversely proportional to the maximum value of $Q$ up to which the reflectivity can be measured. As a rough approximation, to distinguish variations in the scattering length density over a length $L$, the reflectivity must be measured up to a $Q$-value of about $2 \pi / L$.

Another related consideration is the colerence length of the incident neu-. tron, in particular the lateral extent over which its wave front is sufficiently flat. This lateral coherence length depends upon how a neutron incident on the sample was prepared. Imagine the following simplified possibility: suppose that the last interaction a given neutron has, before impinging on and reflecting from a flat thin film, is with a single hydrogen nucleus in some moderator material from which it scatters incoherently and isotropically as a spherical wave with some wavelength, $\lambda$. Subsequently, it reaches the sample along an unobstructed path a distance $S$ away from the hydrogen atom of origin. The radius of the spherical neutron wave is then $S$ and the length $L$ of a line parallel to a tangent to a circle of that radius - but at a distance $S-\lambda$ - is given by

$$
\left(\frac{L}{2}\right)^{2}=\lambda(2 S-\lambda)
$$

The points at either end of this line of length $L$ are a distance $\lambda$ away from the sample surface when the sphere of radius $S$ first makes contact. Thus, these end points define the lateral distance in the sample plane across which a given wave 
front becomes out of phase by $2 \pi$. For a perfectly flat plane wave, this lateral distance is infinite. For a spherical wave of wavelength $5 \AA$ emanating from a source a distance $5 \mathrm{~m}$ away, for example, $L$ is about 140 microns.

Now deviations of the surface from perfect flatness on length scales significantly smaller than the lateral coherence of the incident radiation, as described in the preceding paragraph, give rise to nonspecular scattering. If the nonspecular scattering is small enough compared to the specular component, then the one-dimensional analysis of the specular reflectivity described above is a good approximation: the in-plane roughness will be manifested in the SLD profile by scattering length densities (for slabs of thickness $\mathrm{d} z$ ) which represent average values over the area that the neutron wave is effectively coherent or in phase. If nonspecular scattering is significant, typically more than a few per cent of the specular component, then the analysis of the specular reflectivity as a one-dimensional problem becomes less of a good approximation. If, on the other hand, the sample is composed of in-plane areas with different SLD profiles which have dimensions far larger than the neutron's lateral coherence length, then the observed reflectivity will be proportional to an incoherent sum of independent, weighted reflected intensities for each different region (the weights representing the fractional area of each region). In this latter case, the analysis of the measured reflectivity requires appropriate modelling to account for this effect.

As mentioned earlier, the fact that neutrons can traverse macroscopic distances of single crystalline materials, e.g. silicon, with relatively little attenuation, makes it possible to study films in contact with far less transparent media such as an aqueous reservoir. In this case the single crystal serves not only as the supporting substrate but also as incident medium.

Perhaps neutron scattering's greatest limitation is that the sources are relatively weak compared to what exists for X-rays, for example. It is, therefore, often of crucial importance to maximize sample size and minimize background, primarily by eliminating extraneous scattering material surrounding the sample. Figure 5 illustrates the range of neutron reflectivities which can currently be measured at the NIST Center for Neutron Research for a typical film structure, in this example, $\mathrm{SiO}$ on a silicon substrate. Additional information regarding instrumental resolution and techniques can be found in [2].

\section{Illustrative examples}

Space does not permit an exhaustive review of all of the many applications of neutron reflectometry. Included here are several representative examples and some references to other work.

\subsection{Soft condensed matter}

Probably most of the researchers who have engaged in neutron reflectivity experiments have been interested in studying some form or another of organic film system. Such systems include spin-coated polymers and biological membranes deposited by the Langmuir-Blodgett technique or via self-assembled monolayers. The substrate of choice is most often single crystalline silicon. 
(a)

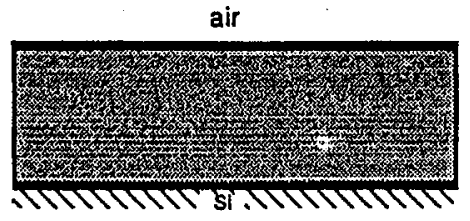

(b)

air

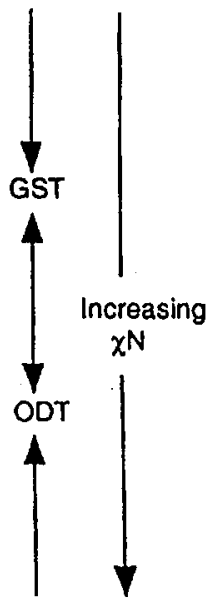

Fig. 6. Thin film morphologies for synmetric PEP/PEE diblock copolymers as discussed in the text (after [9]).

Block copolymers display distinctive microstructures and ordering transitions which are affected by symmetry breaking interfaces. These macromolecules are composed of two distinct monomeric sequences, where each sequence or segment consists of $N$ units of one or the other monomer, linked together at a single point. Neutron reflectometry is an ideal method for studying the thin film structures of such systems if one of the monomeric sequences is deuterated and the other hydrogenated. A comprehensive investigation of this nature was carried out by Foster et al. [9] on symmetric poly(ethylene-propylene)/poly(ethylethylene) (PEP/PEE) diblock copolymers. Figure 6 is a schematic representation of the thin film morphologies of symmetric PEP/PEE diblock copolymers. $\chi$ represents the segment-segment interaction parameter whereas ODT and GST signify order-disorder and Gaussian-stretched coil transitions, respectively. From the reflectivity data of Fig. 7 for various chain lengths, the SLD or concentration profiles normal to the surface of the film shown in Fig. 8 can be deduced. The study clearly demonstrated that the polymer/air and substrate/polymer interfaces can induce an ordered microstructure even when the center of the film is disordered (see Ref. [9] for further discussion and conclusions).

A large body of literature has been published on neutron reflectivity studies of polymer films - two reviews on the subject have been given, by Penfold and Thomas [10] and Russell [11], which are dated but still useful. For more recent work in polymer science as well as in surfactant systems and Langmuir-Blodgett films, the Proceedings of the 4th and 5th International Conferences on Surface X-Ray and Neutron Scattering, [12] and [13], respectively, can be consulted.

Biological membranes can also be studied by neutron reflectivity, either in multilayer or single bilayer form. Figure 9 shows how important information, in this case the overall film thicknesses of the lipid bilayer in the gel and liquid crystal phases, can be obtained with great accuracy [14]. 

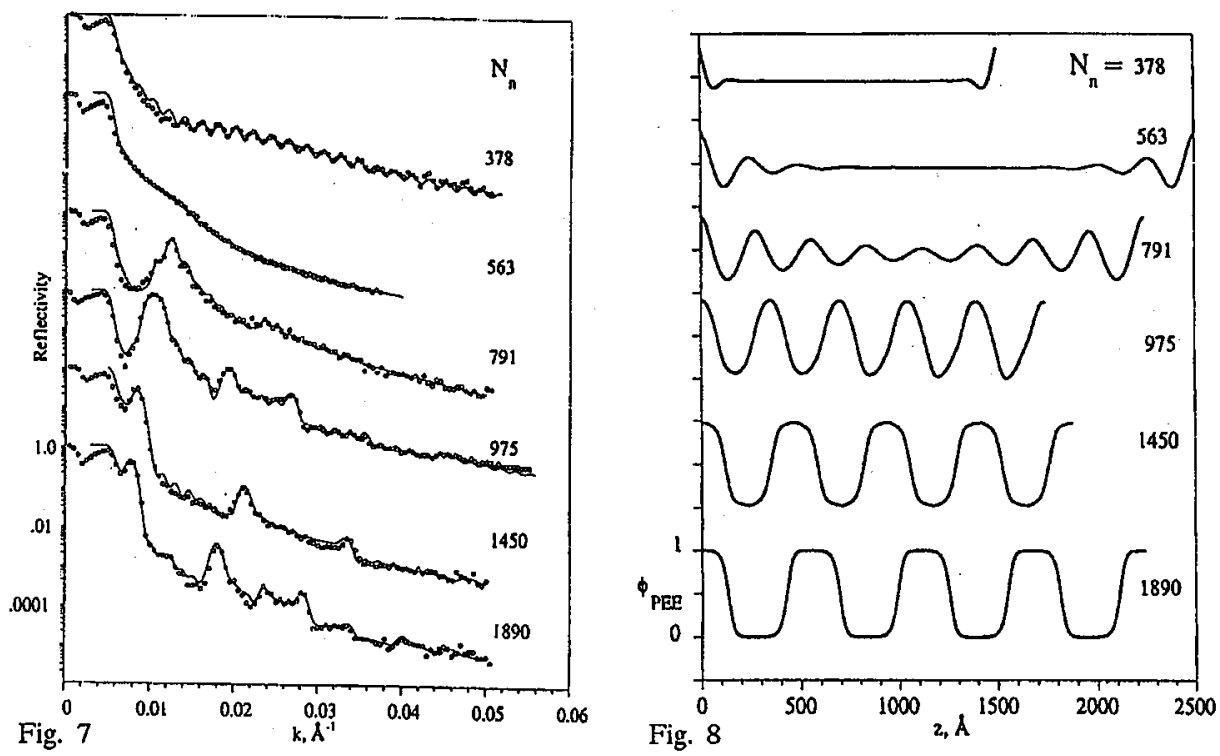

Fig. 7. Neutron reflectivity data for six PEP/PEE chain lengths and the corresponding model curves used to infer the concentration profiles shown in Fig. 8. The results for $N$ less than $\mathbf{1 8 9 0}$ have been shifted vertically. The data below the critical angle for total external reflection has not been corrected for the beam footprint (after [9]).

Fig. 8. Real space concentration profiles corresponding to the model reflectivity curves shown in Fig. 7 (after [9]).

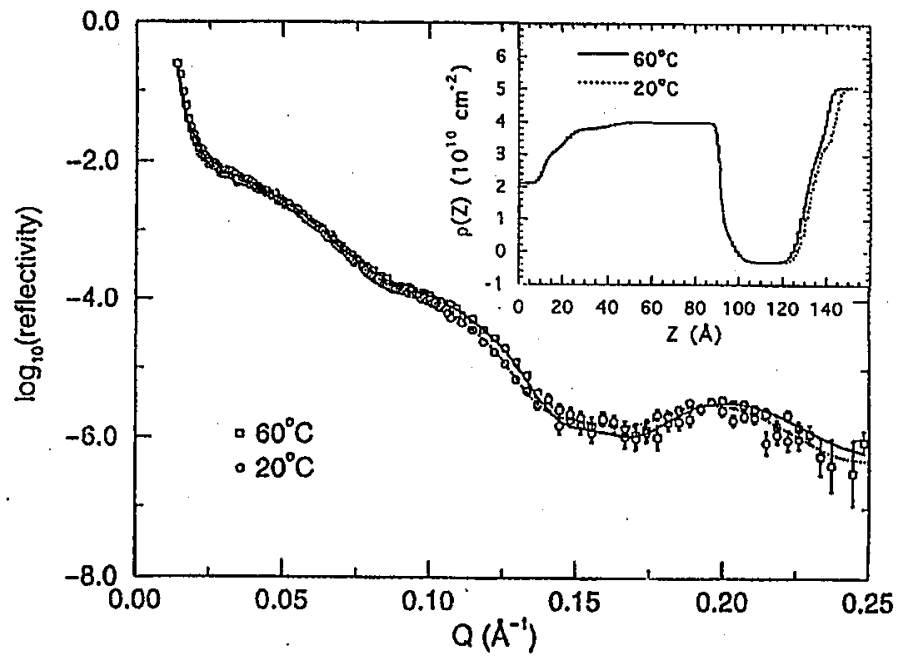

Fig. 9. Neutron reflectivity data and fits corresponding to the two SLD profiles shown in the inset: the sample is a biolayer of PPC/octadecanethiol HBM on $\mathrm{Au}$ in $\mathrm{D}_{2} \mathrm{O}$ (after [14]). 


\subsection{Hard condensed matter}

\subsubsection{Nonmagnetic materials}

Figure 10 shows the neutron reflectivity curves and corresponding SLD profiles obtained for a $\mathrm{Ti} / \mathrm{TiO}$ film, deposited on a $\mathrm{Si}$ substrate, in contact with the aqueous reservoir of an active electrochemical cell [15]. The data show that, with time, the TiO and then the Ti film are corroded away by the electrochemical reaction. Note that the corrosion process is effectively being monitored on a nanometer scale. Figure 11 shows the family of fits which were obtained for one of the reflectivity curves of Fig. 10 using a model-independent procedure [6]. The variations in the SLD profiles, which yield the same goodness-of-fit to the reflectivity curve plotted beneath, are due not only to statistical uncertainties in the measured intensities, but also to the fact that the reflectivity was collected over a finite range of $Q$ and that phase information is missing. The subjects of phase determination

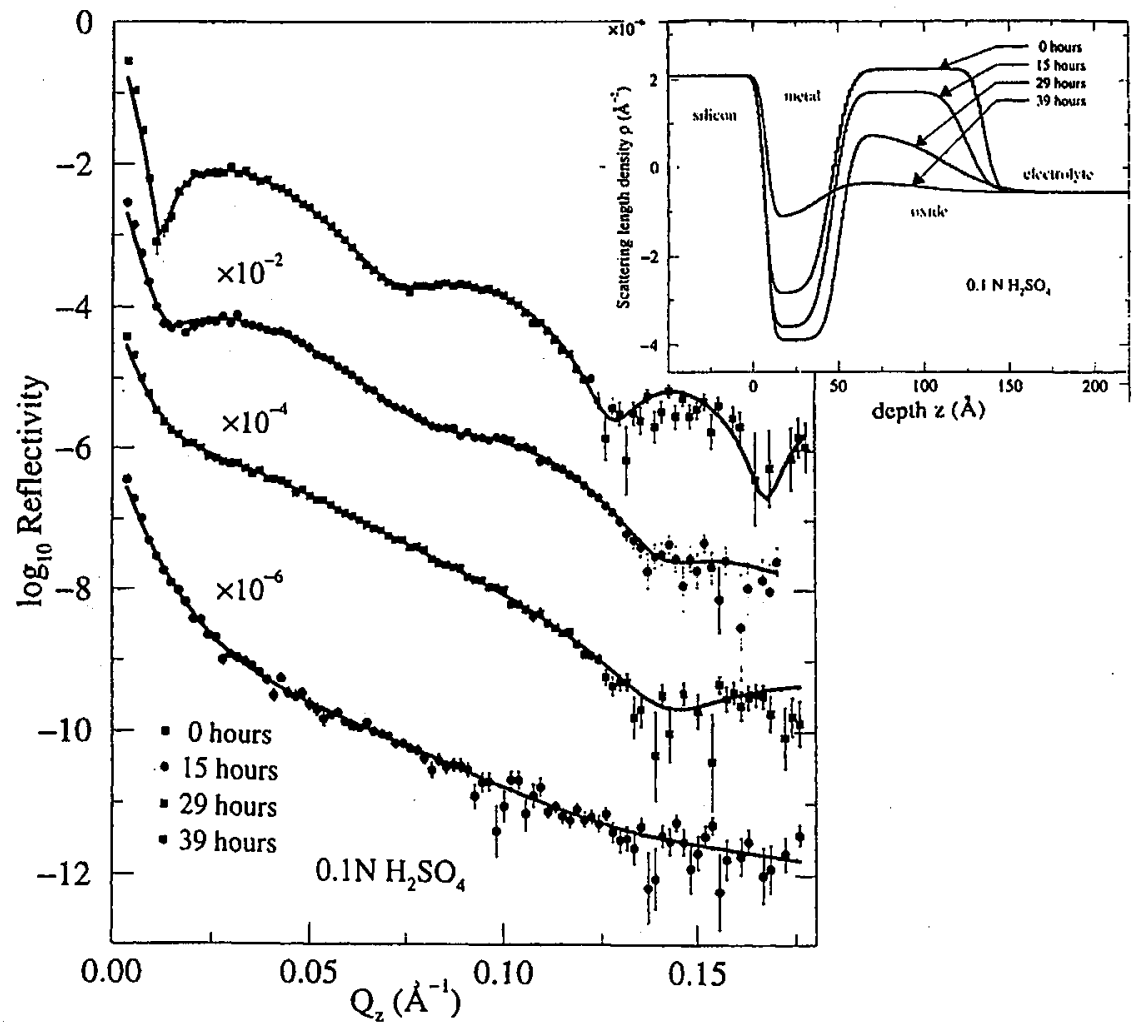

Fig. 10. Titanium/titanium oxide layer SLD profiles and corresponding neutron reflectivity curves as obtained for a specimen in contact with an aqueous solution reservoir of an active electrochemical cell. The corrosion of the layers was studied in situ over the course of about two days (after [15]). 

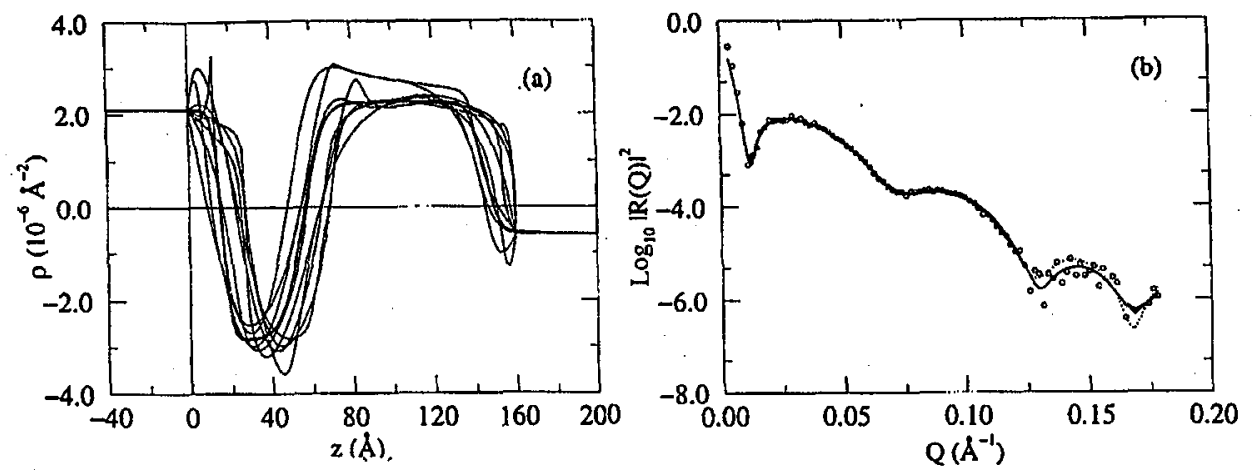

Fig. 11. Family of SLD profiles (a) which give essentially equivalent fits to the reflectivity data (b) (for the Ti/TiO system presented in Fig. 10) (after [6]).

and direct inversion of reflectivity data to obtain unambiguous SLD profiles are discussed in Sec. 5 below.

The diffusion of various isotopes and atoms in solid thin films or into substrates wherein no or relatively little contrast exists for X-rays can be studied by neutron reflectometry. For example, Smith et al. [16] performed a neutron reflectivity study of the self-diffusion of boron using separate layers, each of a single isotope possessing a different neutron scattering length. It was found that standard Fickian diffusion models could not explain the SLD profiles obtained as a function of annealing time.

\subsubsection{Magnetic materials}

It is possible to grow thin magnetic films adjacent to other material layers by a variety of vapor and electrochemical deposition techniques. By constructing synthetic sandwich or multilayered structures, fundamental magnetic phenomena on a microscopic level can be examined by neutron reflectometry in a systematic and controlled manner. More detailed information can generally be extracted from single crystalline superlattices, most often grown by molecular beam epitaxy in an ultra high vacuum environment, since the correlation of an effect with crystallographic orientation can be established. The effects of finite layer thickness, interfaces, and interlayer coupling between magnetic layers separated by nonmagnetic spacers can be quantitatively measured. As discussed in Sec. 2.2, using polarized neutron beams it is possible to obtain the vector magnetization depth profile for a magnetic multilayered structure from neutron reflectivity measurements.

Some of the earliest magnetic superlattices studied by neutron scattering were composed of the rare earths [17]. For example, a series of superlattices made up of alternating single crystalline slabs of Gd and $Y$ were grown, each with the same Gd layer thickness but a different nommagnetic $Y$ layer spacing. Each layer or slab consisted of a well-defined number of atomic planes along the surface normal direction. Because of the superlattice structure, satellite reflections are observed at a period in $Q$ that is inversely proportional to the bilayer thickness. These satellites appear both about $Q$ equal to zero, the reflectivity regime, and about the Bragg peak positions associated with the atomic plane spacings at higher scatter- 
ing angles, the crystal diffraction region. It was found that the ferromagnetic $\mathrm{Gd}$ slabs aligned either parallel or antiparallel to adjacent Gd slabs depending upon the thickness of the intervening $Y$ layer, in an oscillatory manner. A subsequent theoretical explanation of this coupling between Gd layers invoked a long-range, indirect exchange interaction [18]. Similar studies have now been done on a wide variety of magnetic systems, investigating the coupling mechanisms between magnetic layers across normal conducting, superconducting, semiconducting, and insulating layers.

More recently, magnetic thin film systems displaying "giant magnetoresistance" (GMR) behavior have been the subjects of intense interest, both for their fundamental magnetic behavior and techmological importance in the magnetic recording industry. Figure 12 shows both neutron low-angle reflectivity and high-angle diffraction data from which the microscopic structure of an $\mathrm{Fe} / \mathrm{Cr}$ superlattice was deduced [19]. The noncollinear coupling between ferromagnetic slabs across the twisted magnetizations of the $\mathrm{Cr}$ atomic planes (constituting the antiferromagnetic $\mathrm{Cr}$ layers) required a theory which included a biquadratic term in the Fe interlayer coupling potential to explain [20]. Even more exotic physical interactions will probably be required to explain the bizarre nature of the coupling between antiferromagnetic EuTe layers across semiconducting but nonmagnetic $\mathrm{PbTe}$ layers, as discovered by neutron scattering from EuTe/PbTe superlattices [21].

For a review of some of the research on interlayer magnetic coupling and related phenomena using neutron reflectivity and diffraction, see, for example Ref. [22] and other work cited therein.

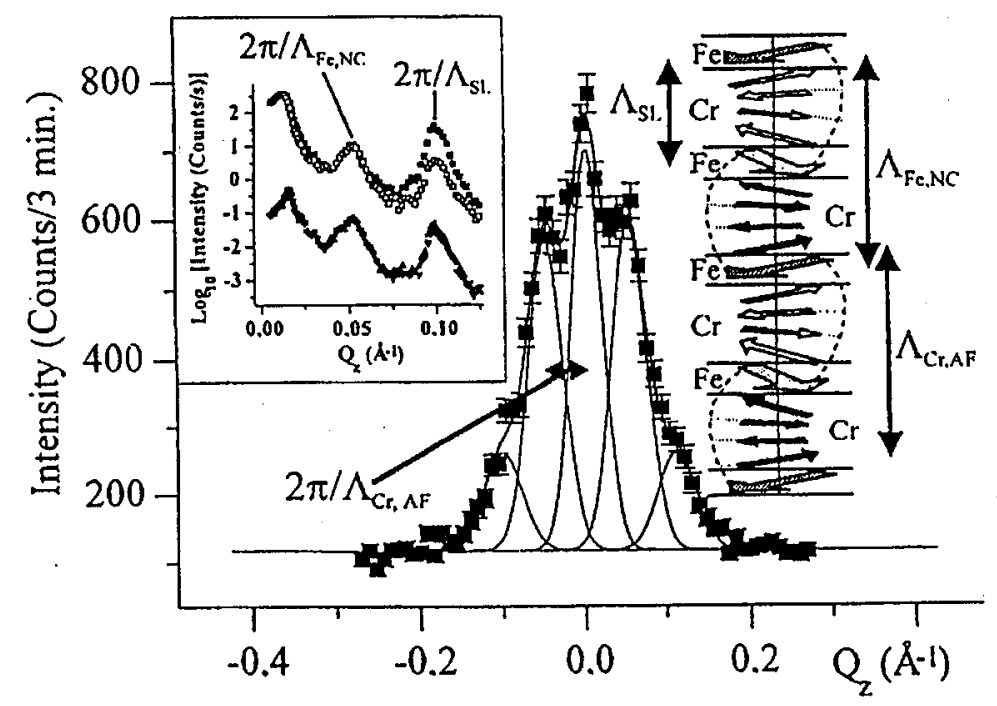

Fig. 12. Low-angle polarized neutron reflectivity curves (left inset) and high-angle neutron diffraction data from which the microscopic magnetic structure of an $\mathrm{Fe} / \mathrm{Cr}$ superlattice, illustrated on the right, was deduced (after [19]). 
It should at least be mentioned here that just as it is possible to measure a magnetization profile normal to the surface of a thin film using polarized neutron reflectometry, the gradual expulsion of an applied magnetic field with depth into a superconducting thin film can be determined. The first such measurement of the magnetic penetration depth was performed by Felcher et al. [23].

\section{Phase determination and inversion}

As discussed in Sec. 2, it is possible to accurately predict the specular reflectivity curve to be expected for a given SLD model using a simple, but powerful, theory based on a one-dimensional wave equation. It is more problematical, however, to extract a SLD profile from a measured set of reflectivity data. Given reflected intensities alone, the only recourse is to use a fitting procedure, which is basically a sophisticated form of trial and error. Even if a good fit to the reflectivity is found, there is no guarantee that it represents the actual SLD profile since a unique solution requires phase information, i.e., the complex reflection amplitucle (the SLD profile of a free-standing film, for example, gives rise to exactly the same reflectivity curve as its mirror image). Figure 13 is an illustration of the ambiguity that can occur in an actual case: the two obviously very different SLD profiles shown were obtained from model-independent fits of the reflectivity with essentially identical goodness of fit (the two curves representing the fits are plotted but are virtually indistinguishable from one another). Realistically, in many situations a nonphysical solution can be ruled out on the basis of additional, independent physical evidence or other constraints on the SLD values. For example, in the study of magnetic thin film systems, the chemical structure can be determined from a combination of neutron and X-ray scattering measurements before performing polarized neutron reflectivity experiments designed to deduce the magnetic architecture. On the other hand, ab initio reflectivity studies of certain film systems, where prior structural and/or compositional information is sufficiently lacking, demand other means of ensuring that a unique SLD is obtained.
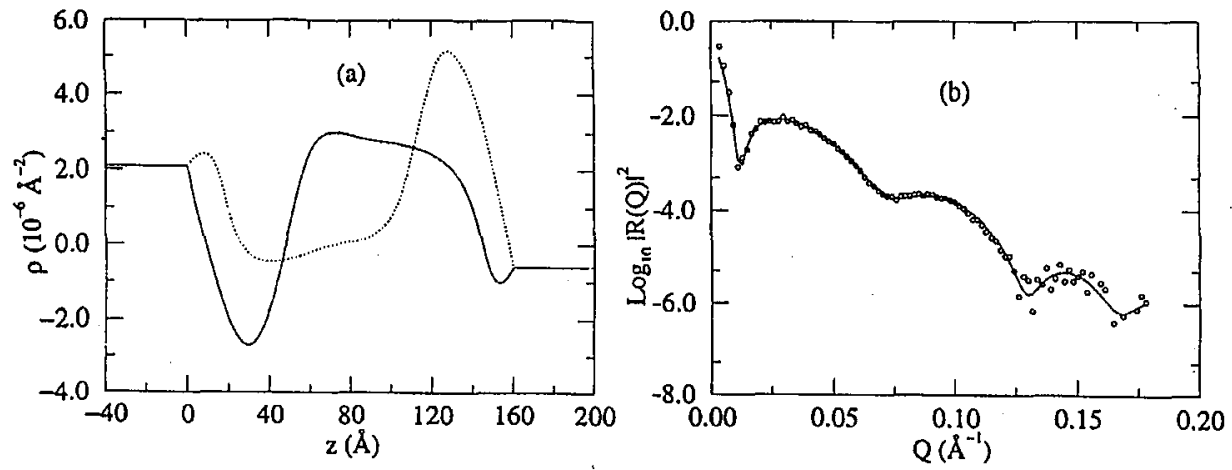

Fig. 13. Demonstration that two symmetry related SLD profiles (a), one physical, the other not, can give essentially identical fits to the measured reflectivity curve (b) (the $\mathrm{Ti} / \mathrm{TiO}$ data presented earlier) in the absence of phase information (after [6]). 
As it turns out, an exact and unique solution of the inverse scattering problem [24], namely, to obtain the SLD profile directly from a scattering pattern, exists for real potentials of finite spatial extent; requirements almost always satisfied for specular neutron reflection. However, the solution methods require that the complex reflection amplitude for the film system of interest be known as a function of $Q$. Fortunately, it is possible to extract this reflection amplitude from measurements of the reflected intensity for two or three composite thin film structures consisting of the "unknown" film adjacent to a reference layer, the SLD profile of which can be altered in a known way $[25,26]$. A convenient choice of a reference layer is a saturated ferromagnetic Fe film deposited on a substrate and buried beneath the sample film being studied. Using polarized neutrons, a beam in the "+" spin state sees one Fe SLD profile, whereas a "_" spin state beam senses an altogether diffe", "It one due to the nature of the interference between the nuclear and magnetic scattering potentials of the Fe. A system of algebraic equations can be written which relate measured reflected intensities for the composite layer systems to combinations of the elements of the transfer matrix described in Sec. 2. In this case, however, the transfer matrix of the composite system can be written as a product of matrices corresponding to the unknown part and the reference in such a way that the two distinct pieces become mathematically separated as well. The system of linear equations which result are exact and apply equally well at all values of $Q$ accessible to experiment, even where the Born approximation,

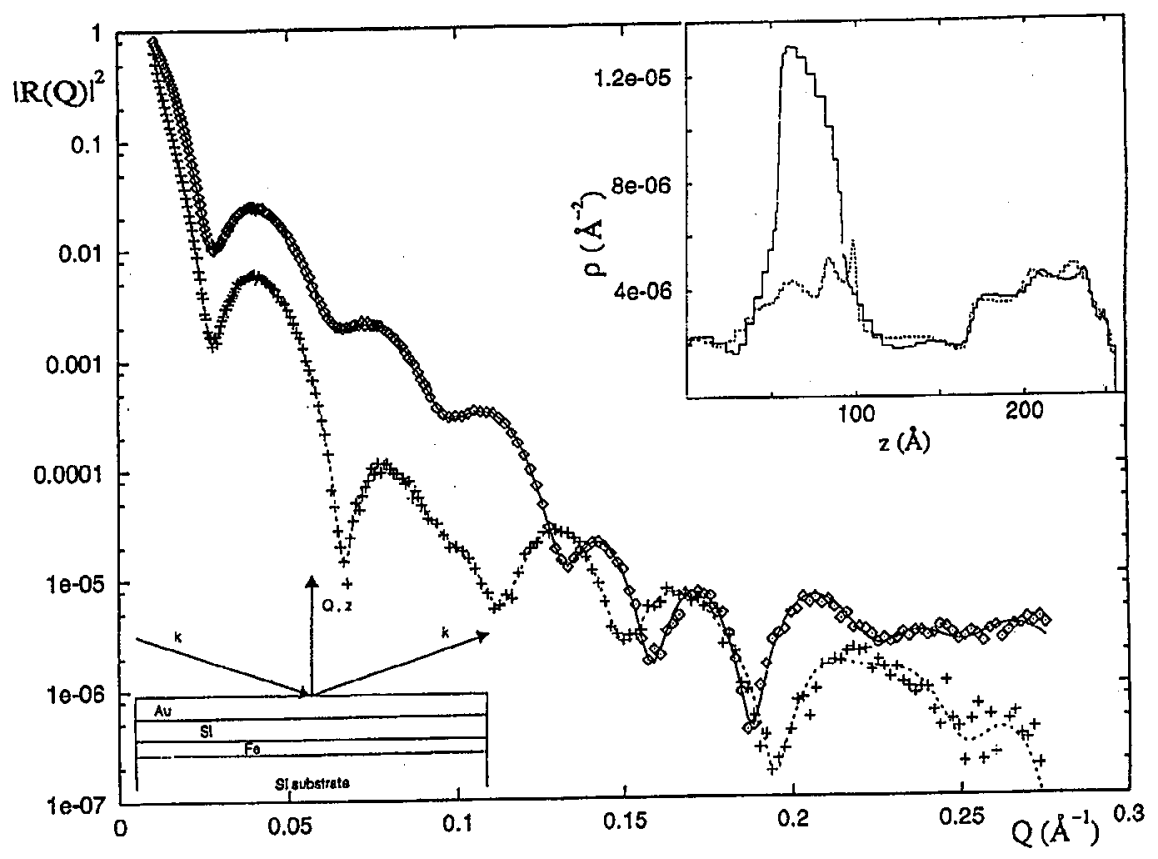

Fig. 14. Neutron reflectivity data and fits corresponding to the SLD profiles shown in the inset for the $\mathrm{Au} / \mathrm{Si} / \mathrm{Fe}$ film sandwich structure discussed in the text. 
as discussed in Sec. 2, is not valid. The solution of these equations then yields the unambiguous value of the complex reflection amplitude for the film of interest alone [25].

Shown in Fig. 14 are the two sets of polarized reflectivity data obtained for the $\mathrm{Fe} / \mathrm{Si} / \mathrm{Au}$ sandwich structure represented in the lower inset. Model independent fits to the data yield the composite SLD profile shown in the upper inset of Fig. 14. As a check of the self-consistency of the phase determination method outlined above, the gold and Si layers were then treated as though they were an "unknown" part of the structure whereas the Fe layer was employed as the reference. Following the phase determination procedure, the imaginary part of the complex reflection amplitude corresponding to the $\mathrm{Au}$ and $\mathrm{Si}$ layers alone was extracted from the same composite reflectivity data sets of Fig. 14 and is plotted in Fig. 15. A specific solution to the one-dimensional inverse scattering problem (based on the method developed by Gel'fand, Levitan, and Marchenko) [27] was subsequently used to retrieve the SLD profile shown in the inset of Fig. 15: this can be seen to be in good agreement with that part of the composite SLD profile of Fig. 14 corresponding to the $\mathrm{Si}$ and Au layers.

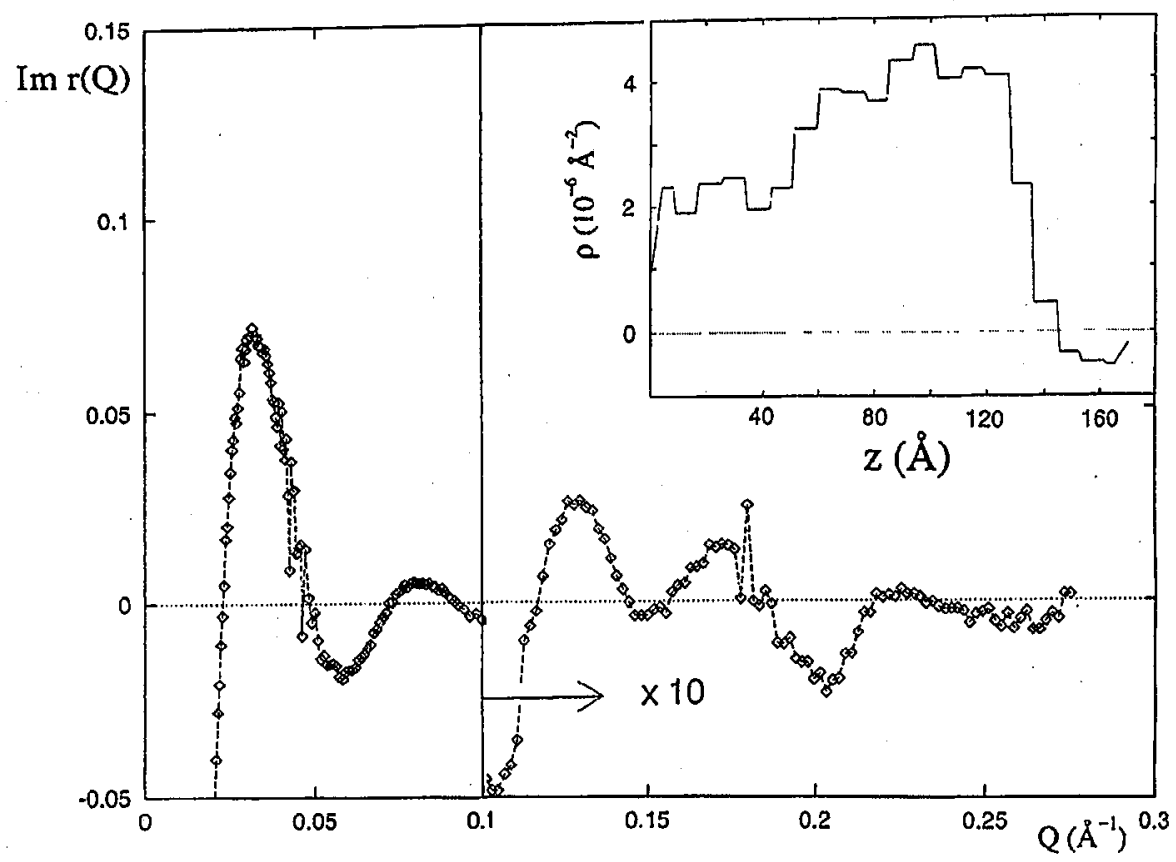

Fig. 15. Imaginary part of the complex reflection amplitude obtained from the reflectivity data of Fig. 14 by taking the Fe film to be a known reference layer: this amplitude then corresponds to the Si and Au layers only. The SLD profile shown in the inset was obtained from a direct inversion of the reflection amplitude based on the integral equation solution of Gel'fand, Levitan, and Marchenko mentioned in the text. The SLD profile for the $\mathrm{Si}$ and $\mathrm{Au}$ layers thus obtained is in good agreement with the appropriate part of that shown in Fig. 14. 
Besides eliminating some of the ambiguity in the SLD profiles extracted from reflectivity data, the reference layer method also has several other advantages. Analysis of model systems indicates that an equivalent degree of spatial resolution in the SLD profile can be obtained for reflection amplitude data collected out to a maximum wave vector transfer approximately only half of that required for reflected intensity data without any phase information. Secondly, a section of the reference structure can be arbitrarily included as part of the unknown. In this way the inversion process itself can be used as a diagnostic: if the incorporated known piece of the potential is not recovered upon inversion, then it can be immediately concluded that the film structure being studied was either inhomogeneous (i.e., incomplete coverage, varying overall thickness, etc.) or sufficiently rough to give rise to a significant amount of nonspecular scattering. For example, in one of the former possibilities, the sample could consist of two or more distinct regions of in-plane dimensions that are large compared to the lateral coherence length of the neutron wave. Then the measured reflectivity will actually consist of a sum of separate, weighted reflected intensities, each corresponding independently to one of the separate regions of the sample. However, this incoherent sum of reflected intensities would not be necessarily evident in the data as collected. In fact, it might very well be possible to obtain a fit to the resultant reflectivity curve that corresponds to a single homogeneous, but completely fictitious, film structure!

Finally, it should be pointed out as well that the SLD profile obtained by inversion using this phase determination method is only as good as the accuracy to which the reference layer structure is known. Furthermore, the reference should be chosen intelligently, in such a way as to optimize sensitivity to the part of the film being studied (this includes "boosting" the signal of a weakly reflecting layer). For instance, it might prove useful to employ a reference with an effectively variable thickness or length rather than scattering length density magnitude only. That is, a reference structure with some element of "frequency" as opposed to "amplitude" modulation might be preferable in certain cases.

\section{Conclusions}

Neutron reflectometry has a wide range of applications in the investigation of the chemical and magnetic nanostructures of thin film materials, including both hard and soft condensed matter. The method complements other scattering probes, including X-rays, electrons, and atomic beams, as well as more local ones such as atomic force and scanning tunneling microscopies.

\section{Acknowledgments}

It is both a responsibility and pleasure to acknowledge all my colleagues, past and present, both at the NCNR and elsewhere, without whose considerable contributions much of the work presented here would not have been possible.

\section{References}

[1] R. Pynn, in: Neutron Scattering in Materials Science II, MRS Symp. Proc., Vol. 376, Eds. D.A. Neumann, T.P. Russell, B.J. Wuensch, MRS, Pittsburgh 1995, p. 165; S.K. Sinha, p. 175. 
[2] C.F. Majkrzak, J.F. Ankner, N.F. Berk, L.D. Gibbs, in: Magnetic Multilayers, Eds. L.H. Bennett, R.E. Watson, World Scientific, Singapore 1994, p. 299.

[3] E. Merzbacher, Quantum Mechanics, 2nd ed., Wiley, New York 1970.

[4] M. Born, E. Wolf, Principles of Optics, 6th ed., Pergamon Press, Oxford 1980, p. 51.

[5] J.F. Ankner, C.F. Majkrzak, in: Neutron Optical Devices and Applications, SPIE Proc., Vol. 1738, Eds. C.F. Majkrzak, J. Wood, SPIE, Bellingham (WA) 1992, p. 260.

[6] N.F. Berk, C.F. Majkrzak, Phys. Rev. B 51, 11296 (1995).

[7] G.E. Bacon, Neutron Diffraction, 3rd ed., Oxford University Press, London 1975.

[8] C.F. Majkrzak, Physica B 213\&214, 904 (1995).

[9] M.D. Foster, M. Sikka, N. Singh, F.S. Bates, S.K. Satija, C.F. Majkrzak, J. Chem. Phys. 06, 8605 (1992).

[10] J. Penfold, R.K. Thomas, J. Phys., Condens. Matter 2, 1369 (1990).

[11] T.P. Russell, Mater. Sci. Rep. 5, 171 (1990).

[12] Proc. 4th Int. Conf. Surface X-Ray and Neutron Scattering, Eds. G.P. Felcher, H. You, Physica B 221 (1996).

[13] Proc. 5th Int. Conf. Surface X-Ray and Neutron Scattering, Eds. D. Norman, J.R.P. Webster, Physica B 248 (1998).

[14] C.W. Meuse, S. Krueger, C.F. Majkrzak, J.A. Dura, J. Fu, J.T. Connor, A. Plant, Biophys. J. 74, 1388 (1998).

[15] D.W. Wiesler, C.F. Majkrzak, Physica B 198, 181 (1994).

[16] G.S. Smith, W. Hamilton, M. Fitzsimmons, S.M. Baker, K.M. Hibbard, M. Nastasi, J-P. Hirvonen, T.G. Zocco, in: Neutron Optical Devices and Applications, SPIE Proc., Vol. 1738, Eds. C.F. Majkrzak, J. Wood, SPIE, Bellingham (WA) 1992, p. 246.

[17] C.F. Majkrzak, J. Kwo, M. Hong, Y. Yafet, D. Gibbs, C.L. Chien, J. Bohr, Adv. Phys. 40, 99 (1991).

[18] Y. Yafet, J. Appl. Phys. 61, 4058 (1987).

[19] A. Schreyer, C.F. Majkrzak, Th. Zeidler, T. Schmitte, P. Bodeker, K. Theis-Brohl, A. Abromeit, J.A. Dura, T. Watanabe, Phys. Rev. Lett. 79, 4914 (1997).

[20] J.C. Slonczewski, J. Appl. Phys. 73, 5957 (1993).

[21] K.I. Goldman, G. Springholz, H. Kepa, T.M. Giebultowicz, C.F. Majkrzak, G. Bauer, Physica B 241-243, 710 (1998).

[22] C.F. Majkrzak, Physica B 221, 342 (1996).

[23] G.P. Felcher, R.T. Kampwirth, K.E. Gray, R. Felici, Phys. Rev. Lett. 52, 1539 (1984).

[24] K. Chadan, P.C. Sabatier, Inverse Problems in Quantum Scattering Theory, 2nd ed., Springer, New York 1989.

[25] C.F. Majkrzak, N.F. Berk, Phys. Rev. B 52, 10827 (1995).

[26] V.O. deHaan, A.A. van Well, S. Adenwalla, G.P. Felcher, Phys. Rev. B 52, 10831 (1995).

[27] N.F. Berk, C.F. Majkrzak, J. Phys. Soc. Jpn. 65, 107 (1996). 